\title{
Evaluation of strokes in children
}

\author{
Dimuthu Saraji Wijesekara \\ Senior Registrar in Paediatric Neurology, Lady Ridgeway Hospital, Colombo.
}

\section{Introduction}

Childhood stroke is perceived to be relatively rare. Epidemiological studies have revealed an annual incidence of approximately 3 per 100,000 paediatric strokes in children above one month of age ${ }^{1}$. Most of the strokes are ischaemic in origin while about $40-50 \%$ childhood strokes are of hemorrhagic in origin ( $\mathrm{ICH}$, Subarachnoid haemorrhages), although these figures vary in different studies ${ }^{2}$. Arterial ischaemic strokes (AIS) and venous ischaemia are the types of ischaemic strokes that occur in children. Some studies suggest that venous ischaemia is more common.

\section{Clinical presentation}

The signs and symptoms depend on the location and size of the occluded vessel as well as the patient's age. Anterior circulation strokes are much more common than posterior circulation strokes 1. Two-thirds of children will present with an acute face-arm-leg weakness ${ }^{1}$. Seizures or altered level of consciousness may complicate presentation. Preceding self-limited episodes of hemiparesis are experienced in $20 \%$ of patients 1 .

Infants may have no clinical manifestations or delayed clinical presentation with initially flaccid paralysis in one side and later becoming spastic. Pathologic early hand preference is commonly a presenting complaint in infants. Firstly it may be that the occlusion is gradual, allowing adequate arterial anastomotic channels to develop. In addition, the potential for recovery is so great that children may be seen without major deficit for months or years after an ischemic event, despite large areas of brain infarction. A third reason for the delay in clinical presentation in infants is that identification of clinical signs may not occur until brain maturation reaches a stage allowing expression of the deficit ${ }^{3}$.

\section{Aetiology of stroke in children}

In adults, the common underlying risk factors for stroke include hypertension, diabetes mellitus, atherosclerosis, cardiac arrhythmias and valvular abnormalities ${ }^{4}$. In childhood, however, the potential aetiologies are many which create a greater diagnostic challenge to the clinician.

Cardio-embolic causes are the most frequent cause of paediatric strokes. Potential mechanisms of stroke in cyanotic congenital heart disease include hyperviscosity and diminished oxygenation of blood, paradoxical emboli from right-to-left shunting and emboli from vegetations secondary to valvular disease. In the context of developing countries as in Sri Lanka, valvular lesions from acquired rheumatic heart disease play a major role. Other abnormal structural defects predisposing to emboli include atrial myxoma, cardiac rhabdomyoma, cardiomyopathies, bacterial endocarditis, and prosthetic valves. Cardiac arrhythmias, particularly atrial fibrillation, as in adults, predispose to emboli.

A variety of haematological causes will lead to arterial ischemic disease in children, though venous occlusion and hemorrhagic events may also occur in the same disease processes. Hyperviscosity syndromes (polycythemia, hyperleukocytosis as in leukaemias, and thrombocytosis) can lead to occlusion of vessels ${ }^{5}$. Haemoglobinopathies, the prototype of which is sickle cell disease, are often complicated by stroke. The incidence of stroke in sickle cell disease is between $5 \%$ and $10 \%$, with the median age of the first stroke being seven years of age. Recurrence without treatment occurs in up to $90 \%$ of patients, most within three years ${ }^{6}$.

There has been a recent recognition of the importance of hypercoagulable states. These may be genetically acquired, associated with autoimmune and other systemic disorders, or found independent of an underlying disease. Anti Thrombin III, protein $\mathrm{C}$, and protein $\mathrm{S}$ are 
naturally occurring anticoagulants whose deficiencies are inherited as autosomal recessive traits $^{7}$. Autoimmune disorders may lead to cerebrovascular disease through a vasculitis or by inducing a hypercoagulable state. A hypercoagulable state is created by antiphospholipid antibodies, which includes the lupus anticoagulant and the anti-cardiolipin antibody $^{8}$. In systemic lupus erythematosis, neurologic involvement is seen in over $50 \%$ of patients. Prothombin gene mutation (G20210A), factor $\mathrm{V}$ laiden mutation and hyperhomocystinaemia have also been attributed to the causation of childhood stroke ${ }^{9}$. Homocystinuria, due to defect of methionine metabolism, may present as a thrombotic syndrome. High levels of homocystine lead to endothelial damage and increased platelet aggregation. Young adults heterozygous for homocystinuria are also felt to be at increased risk ${ }^{10}$.

Polyarteritis nodosa, Wegener's granulomatosis, Henoch-Schonlein purpura, ulcerative colitis, Kawasaki disease and the dermatomyositis / polymyositis complex are rare associations with childhood stroke due to associated arteriopathies ${ }^{11}$.

Stroke-like episodes in a nonvascular distribution are seen in mitochondrial encephalomyopathy with lactic acidaemia and stroke-like episodes (MELAS). This disorder is due to a mutation of mitochondrial DNA and may require muscle biopsy to confirm.

In one third of thrombotic carotid artery occlusion, a preceding infection is noted, often in the pharynx or cervical area. Pharyngitis, cervical adenitis, tonsillitis, sinusitis and retropharyngeal abscess have all been reported to be precursors of internal carotid artery thrombosis. The mechanism is likely to be local inflammation of the arterial wall.

Cat-scratch fever, mycoplasma, and viral encephalitis have also been associated with vasculopathies causing stroke in children and varicella vasculopathy is described more often in children than others ${ }^{12}$.

Trauma to the neck may predispose to carotid thrombosis. This can be a blunt injury to the neck, intraoral trauma, i.e. falling with a pencil in the mouth, or trauma to the cervical spine. Carotid dissection should always be considered as a cause of stroke. It may be spontaneous or follow a neck injury. In a dissection, a tear in the arterial wall leads to an expanding haematoma which obstructs blood flow. The patient may complain of neck pain or referred pain to the eye or forehead.

Various drugs, illicit and legally prescribed, have been linked to strokes. Cocaine, phencyclidine (PCP), and amphetamines predispose to vascular injury via hypertension, vasospasm and vasculitis. Steroids may cause endothelial hyperplasia and increase platelet adhesiveness.

In young girls the intake of oral contraceptive pills may also contribute by way of creating a hypercoagulable state predisposing to a state of cerebral venous sinus thrombosis.

Moyamoya disease is another rare cause of late childhood stroke which is characterized by an angiographic picture of occlusion of internal carotid arteries and the development of a fine web-like collection of abnormal anastomotic vessels (puff of smoke) ${ }^{13}$.

Migraine-related stroke has been reported. Diagnosis of migraine-related stroke is suggested by a family history of migraine, prior migraines in the patient, and exclusion of other aetiologies by a thorough diagnostic evaluation ${ }^{14}$.

Approximately a quarter to one third of children with strokes, have no recognizable cause. It may be that the clinical knowledge, the expertise in interpreting investigation results and the unavailability of high tech investigations on childhood stroke is not adequate.

In addition to the ischaemic strokes the causes for hemorrhagic strokes include arteriovenous malformations and aneurysms. Bleeding diathesis (thrombocytopenia, leukaemia, sickle cell disease and coagulopathies) and rarely intracranial tumours could also be incriminated.

\section{Differential Diagnosis}

Focal cerebral ischaemia

Intracranial haemorrhage

Cerebral abscess

Encephalitis (herpes simplex virus)

Brain tumor

Multiple sclerosis

Epilepsy: post-ictal Todd's paralysis or a focal inhibitory seizure

Complicated migraine 
Once the diagnosis of a stroke is determined the cause for the stroke needs to be evaluated. This may be a challenging task for the clinician.

It is always important to obtain a very good history regarding the onset, progression and the disabilities, immediate or recent events preceding the stroke, a detailed birth and a developmental history, past medical history of similar illnesses or any cardiac disease and migraine, family history of premature atherosclerosis, heart disease and of unexplained thrombotic episodes and migraine.

A social history should include the sexual history, drug abuse and engagement in body contact sports like rugby and karate.

A detailed general examination with regard to dysmorphism, neurocutaneous stigmata and evidence of connective tissue disorders like butterfly rash, palpable purpura etc.
It is necessary to auscultate the neck and skull for any bruits.

A good cardiovascular system examination and a very detailed neurological examination is a must to detect clues to the diagnosis.

Once the history and the examination are completed, an array of investigations waiting in order to aid in detecting the cause. There are mandatory preliminary investigations that need to be carried out and if the preliminary investigations fail to detect a cause, it is necessary to go onto the second and third line investigations. However, still there could be about a third where the cause is unknown.

\section{Investigations}

\section{$\underline{1}^{\text {st }}$ Line}

CT scan of brain

MRI of brain /MR angiogram

\section{/MRV}

Complete blood count

ESR

BUN, creatinine

Urinalysis

Random glucose

Liver function tests

PT/APTT

$\mathrm{SE}, \mathrm{Ca}, \mathrm{Mg}$,

Chest x-ray

ANA

Echocardiogram

(transthoracic) with saline

contrast

12-lead ECG $\underline{2}^{\text {nd }}$ Line

Holter monitor

Transcranial and / or carotid dopplers

Cerebral angiogram

(transfemoral)

EEG

Antithrombin III

Protein C (activity and

antigen)

Factor V (laiden) mutation

Antiphospholipid antibody

Anticardiolipin antibody

Lupus-anticoagulant

fibrinogen levels -

qualitative/quantitative

Factor viii levels

\section{$\underline{3^{r d} \text { Line }}$}

Urine for organic acids

Hemoglobin electrophoresis

Complement profile

VDRL

Lactate / pyruvate

Ammonia

CSF: cell count, protein,

glucose, lactate

PCR TB

Blood culture

Lipid profile

HIV

Mycoplasma titers

Echocardiogram

(transesophageal)

Muscle Biopsy

DNA testing for MELAS

Leptomeningeal biopsy

Serum homocystine after

methionine load

Genetic studies 


\section{Management}

The acute treatment of cerebral ischemia is largely supportive and requires an intensive care setting. Attention to oxygenation, fluid and electrolyte status, seizures, and infections are critical. Treatment should be directed to the underlying cause if it is identifiable.

Cerebral oedema is maximal over the first 72 hours. Initially, oedema is cytotoxic, although a vasogenic component occurs after two to three days. Edema is usually effectively managed with hyperventilation and fluid restriction. In general, the use of steroids and osmotic agents are not indicated. However, in case of progressive deterioration, mannitol and cranial flap removal may be life saving ${ }^{16}$.

The use of anticoagulation in pediatric ischemic stroke is controversial, although it is often used in the presence of a definable and recurrent source of emboli or evolving thrombotic stroke 15. Anticoagulation is contraindicated in hemorrhagic infarct and uncontrolled hypertension. Long-term anticoagulation with warfarin is indicated in deficiency of protein $\mathrm{C}, \mathrm{S}$, antithrombin III and in the presence of antiphospholipid antibodies. Rehabilitation through aggressive physical, occupational, and speech therapy is essential for all patients. Behavioral problems and learning disabilities may become apparent upon returning to school. Children may need neurocognitive testing and special educational classes ${ }^{17}$.

\section{Prognosis}

The prognosis for childhood strokes is variable. With aggressive rehabilitation most would be near normal in activities of daily living when compared with adults.

\section{References}

1. Schoenberg BS, Mellinger JF, Schoenberg DG. Cerebrovascular disease in infants and children: A study of incidence, clinical features, and survival. Neurology 1978; 28: 763-8.

2. FJ Kirkham. Stroke in children. Arch Dis Child 1999; 81: 85-9.
3. Abram HS. Vascular Disease. In David RB (ed). Child and Adolescent Neurology. Mosby, St. Louis, 1998: 271-93.

4. C Hajat, E Wraige, C Wolfe, V Ganesan. Comparison of the aetiology of ischaemic stroke in children and young adults (United Kingdom) King's College London (Stroke Unit).

5. Tabori U, Beni-Adani L, Dvir R, et al. Risk of venous thromboembolism in pediatric patients with brain tumors. Pediatr Blood Cancer. 2004; 43: 633-6.

6. Balkaran B, Char G, Morris JS, Thomas PW, Serjeant BE, Serjeant GR. Stroke in a cohort of patients with homozygous sickle cell disease. J Paed 1992; 120: 360-6.

7. Sebire Guillaume, Fullerton Heather, Riou Emilie, de Veber Gabrielle. Toward the definition of cerebral arteriopathies of childhood. Current Opinion in Pediatrics 2004: 16(6); 617-22.

8. Miyakis S, Lockshin MD, Atsumi $\mathrm{T}$, et al International consensus statement on an update of the classification criteria for definite antiphospholipid syndrome (APS). J Thromb Haemost 2006; 4: 295306.

9. David R Trevarthen. The Role of Hypercoagulable States in Stroke. Stroke 2000; 11(2).

10. David Green. Thrombosis and stroke, topics in stroke rehabilitation. 2003: 10(3):

11. Pabinger I, Schneider B. Thrombotic risk in hereditary antithrombin III, protein C, or protein S deficiency. A cooperative, retrospective study. Gesellschaft fur Thrombose- und Hamostaseforschung (GTH) Study Group on Natural Inhibitors. Arterioscler Thromb Vasc Biol. 1996; 16: 742-8.

12. Girija AS, Rafeeque M, Abdurehman KP Neurological complications of chickenpox. Annals of Indian Academy of Neurology 2007; 10(4): 240-6.

13. Roy Sucholeiki, MD Moyamoya Disease. eMedicine (last updated) Nov 2006

14. Broderick JP, Swanson JW. Migraine-related strokes: Clinical profile and prognosis in 20 patients. Arch Neurol 1987; 44: 868-71.

15. Fenella J Kirkham. Stroke and cerebrovascular disease in childhood, Current Paediatrics 2003; 13(5): 350-9.

16. Stroke and Neurovascular Disorders, Investigation and Management. Clinical Guideline. Great Ormond Street Hospital UK.

17. C Barnes, F Newall, J Furmedge, M Mackay, P Monagle. Arterial ischaemic stroke in children. Journal of Paediatrics and Child Health 2004: 40(7): 384-7. 